\title{
Real-Time Reverse Transcription-Polymerase Chain Reaction Assay for SARS-associated Coronavirus
}

\author{
Shannon L. Emery, ${ }^{*}$ Dean D. Erdman, ${ }^{*}$ Michael D. Bowen, ${ }^{*}$ Bruce R. Newton, ${ }^{*}$ Jonas M. Winchell, \\ Richard F. Meyer, ${ }^{*}$ Suxiang Tong, ${ }^{*}$ Byron T. Cook, ${ }^{*}$ Brian P. Holloway, ${ }^{*}$ Karen A. McCaustland, ${ }^{*}$ \\ Paul A. Rota, ${ }^{*}$ Bettina Bankamp, ${ }^{*}$ Luis E. Lowe, ${ }^{*}$ Tom G. Ksiazek, ${ }^{*}$ William J. Bellini, ${ }^{*}$ \\ and Larry J. Anderson*
}

\begin{abstract}
A real-time reverse transcription-polymerase chain reaction (RT-PCR) assay was developed to rapidly detect the severe acute respiratory syndrome-associated coronavirus (SARS-CoV). The assay, based on multiple primer and probe sets located in different regions of the SARSCoV genome, could discriminate SARS-CoV from other human and animal coronaviruses with a potential detection limit of $<10$ genomic copies per reaction. The real-time RTPCR assay was more sensitive than a conventional RTPCR assay or culture isolation and proved suitable to detect SARS-CoV in clinical specimens. Application of this assay will aid in diagnosing SARS-CoV infection.
\end{abstract}

Tn late 2002, a life-threatening febrile respiratory illness appeared in Guangdong Province, China, and quickly spread throughout Asia and to other parts of the world (1-4). Designated "severe acute respiratory syndrome" (SARS), the etiologic agent was later identified as a hitherto unrecognized coronavirus (SARS-CoV) $(5,6)$. A diagnosis of SARS is based primarily on clinical and epidemiologic criteria, but many respiratory viruses can cause similar symptoms, and therefore rapid, reliable diagnostic tests for SARS-CoV infection were needed. In response to this need, three types of diagnostic tests for SARS-CoV were quickly developed: tissue culture isolation, antibody detection, and reverse transcription-polymerase chain reaction (RT-PCR) assays.

A variety of RT-PCR assays were developed during the epidemic for SARS-CoV (1,5-8), including a commercial ready-to-use RT-PCR kit (Artus Biotech, Hamburg, Germany). Early RT-PCR assays based on conventional designs required postamplification product processing

${ }^{*}$ Centers for Disease Control and Prevention, Atlanta, Georgia, USA (e.g., gel electrophoresis), were time-consuming, and were prone to false-positive results from amplicon contamination. Conversely, real-time RT-PCR assays based on detecting and quantifying a fluorescent signal generated during amplification do not require postamplification processing and therefore eliminate one potential avenue for template contamination.

A variant of the real-time format, based on TaqMan probe hydrolysis technology (Applied Biosystems, Foster City, CA), has been shown to provide sensitive, specific, and quantifiable results in viral diagnostic assays (9) and has been used successfully to study emerging virus infections $(10,11)$, including SARS $(6,12)$. In response to the SARS public health emergency, we developed and evaluated a TaqMan real-time RT-PCR assay based on three distinct targets in the SARS-CoV genome for rapid deployment to the National Laboratory Response Network for Bioterrorism (LRN) (http://www.cdc.gov/programs/bio.htm).

\section{Materials and Methods}

\section{Clinical Specimens}

A total of 340 clinical specimens collected from 246 persons with confirmed or suspected SARS-CoV infection (13) were used in this study. Specimens included oroand nasopharyngeal swabs (dry and in viral transport media), sputa, nasal aspirates and washes, bronchoalveolar lavage, and lung tissue specimens collected at autopsy. Specimen processing was performed in a class II biological safety cabinet using biosafety level three (BSL3) work practices. Three $100-\mu \mathrm{L}$ aliquots of each specimen were distributed to vials each containing $900 \mu \mathrm{L}$ of NucliSens lysis buffer (bioMérieux, Durham, NC) and stored at $-70^{\circ} \mathrm{C}$ until testing. 


\section{Virus Culture}

Vero E6 cells were inoculated with clinical specimens and observed for cytopathic effect, consisting of cell rounding with a refractive appearance followed by detachment from the flask surface (5). Plaque titrations were conducted by standard methods (14).

\section{Nucleic Acid Extraction}

Nucleic acids were recovered from clinical specimens using the automated NucliSens extraction system (bioMérieux). Following manufacturer's instructions, specimens received in NucliSens lysis buffer were incubated at $37^{\circ} \mathrm{C}$ for $30 \mathrm{~min}$ with intermittent mixing, and $50 \mu \mathrm{L}$ of silica suspension, provided in the extraction kit, was added and mixed. The contents of the tube were then transferred to a nucleic acid extraction cartridge and processed on an extractor workstation. Approximately $40-50 \mu \mathrm{L}$ of total nucleic acid eluate was recovered into nuclease-free vials and either tested immediately or stored at $-70^{\circ} \mathrm{C}$.

\section{Primers and Probes}

Multiple primer and probe sets were designed from the Urbani strain of SARS-CoV polymerase $1 \mathrm{~b}$ and nucleocapsid gene sequences (15) by using Primer Express software version 1.5 or 2.0.0 (Applied Biosystems) with the following default settings: primer melting temperature $\left(\mathrm{T}_{\mathrm{M}}\right)$ set at $60^{\circ} \mathrm{C}$; probe $\mathrm{T}_{\mathrm{M}}$ set at $10^{\circ} \mathrm{C}$ greater than the primers at approximately $70^{\circ} \mathrm{C}$; and no guanidine residues permitted at the $5^{\prime}$ probe termini. All primers and probes were synthesized by standard phosphoramidite chemistry techniques at the Biotechnology Core Facility at the Centers for Disease Control and Prevention (CDC).
TaqMan probes were labeled at the $5^{\prime}$-end with the reporter molecule 6-carboxy-fluorescein (FAM) and at the 3'-end with the quencher Blackhole Quencher 1 (Biosearch Technologies, Inc., Novato, CA). Optimal primer and probe concentrations were determined by cross-titration of serial twofold dilutions of each primer against a constant amount of purified SARS-CoV RNA. Primer and probe concentrations that gave the highest amplification efficiencies in this study were selected for further study (Table 1).

\section{Real-Time RT-PCR Assay}

The real-time RT-PCR assay was performed by using the Real-Time One-Step RT-PCR Master Mix (Applied Biosystems). Each $25-\mu \mathrm{L}$ reaction mixture contained $12.5 \mu \mathrm{L}$ of $2 \mathrm{X}$ Master Mix, $0.625 \mu \mathrm{L}$ of the $40 \mathrm{X}$ MultiScribe and RNase Inhibitor mix, $0.25 \mu \mathrm{L}$ of $10 \mu \mathrm{M}$ probe, $0.25 \mu \mathrm{L}$ each of $50 \mu \mathrm{M}$ forward and reverse primers, $6.125 \mu \mathrm{L}$ of nuclease-free water, and $5 \mu \mathrm{L}$ of nucleic acid extract. Amplification was carried out in 96well plates on an iCycler iQ Real-Time Detection System (Bio-Rad, Hercules, CA). Thermocycling conditions consisted of $30 \mathrm{~min}$ at $48^{\circ} \mathrm{C}$ for reverse transcription, $10 \mathrm{~min}$ at $95^{\circ} \mathrm{C}$ for activation of the AmpliTaq Gold DNA polymerase, and 45 cycles of $15 \mathrm{~s}$ at $95^{\circ} \mathrm{C}$ and $1 \mathrm{~min}$ at $60^{\circ} \mathrm{C}$. Each run included one SARS-CoV genomic template control and at least two no-template controls for the extraction (to check for contamination during sample processing) and one no-template control for the PCR-amplification step. As a control for PCR inhibitors, and to monitor nucleic acid extraction efficiency, each sample was tested by real-time RT-PCR for the presence of the human ribonuclease (RNase) P gene (GenBank accesssion number NM

\begin{tabular}{|c|c|c|c|c|}
\hline Assay ID & Primer/ probe & Sequence $\left(5^{\prime}>3^{\prime}\right)$ & Genomic region & Location $^{\mathrm{b}}$ \\
\hline \multicolumn{5}{|c|}{ Primary diagnostic assay } \\
\hline \multirow[t]{3}{*}{ SARS1 } & $\mathrm{F}$ & CAT GTG TGG CGG CTC ACT ATA T & RNA polymerase & $15370-15392$ \\
\hline & $\mathrm{R}$ & GAC ACT ATT AGC ATA AGC AGT TGT AGC A & & $15422-15449$ \\
\hline & $\mathrm{P}$ & TTA AAC CAG GTG GAA CAT CAT CCG GTG & & $15395-15420$ \\
\hline \multirow[t]{3}{*}{ SARS2 } & $\mathrm{F}$ & GGA GCC TTG AAT ACA CCC AAA G & Nucleocapsid & 28531-28552 \\
\hline & $\mathrm{R}$ & GCA CGG TGG CAG CAT TG & & 28581-28597 \\
\hline & $\mathrm{P}$ & CCA CAT TGG CAC CCG CAA TCC & & 28559-28574 \\
\hline \multirow[t]{3}{*}{ SARS3 } & $\mathrm{F}$ & CAA ACA TTG GCC GCA AAT T & Nucleocapsid & 29016-29034 \\
\hline & $\mathrm{R}$ & CAA TGC GTG ACA TTC CAA AGA & & 29063-29083 \\
\hline & $\mathrm{P}$ & CAC AAT TTG CTC CAA GTG CCT CTG CA & & 29036-29061 \\
\hline \multicolumn{5}{|c|}{ To confirm positive results } \\
\hline \multirow[t]{3}{*}{ N3 } & $\mathrm{F}$ & GAA GTA CCA TCT GGG GCT GAG & Nucleocapsid & $28432-28452$ \\
\hline & $\mathrm{R}$ & CCG AAG AGC TAC CCG ACG & & $28383-28400$ \\
\hline & $\mathrm{P}$ & CTC TTT CAT TTT GCC GTC ACC ACC AC & & 28406-28431 \\
\hline \multirow[t]{3}{*}{$3^{\prime} \mathrm{NTR}$} & $\mathrm{F}$ & AGC TCT CCC TAG CAT TAT TCA CTG & $3^{\prime}$ nontranslated region & 29619-29642 \\
\hline & $\mathrm{R}$ & CAC CAC ATT TTC ATC GAG GC & & 29576-29595 \\
\hline & $\mathrm{P}$ & TAC CCT CGA TCG TAC TCC GCG T & & $29597-29618$ \\
\hline \multirow[t]{3}{*}{ M } & $\mathrm{F}$ & TGT AGG CAC TGA TTC AGG TTT TG & Membrane protein & $26951-26973$ \\
\hline & $\mathrm{R}$ & CGG CGT GGT CTG TAT TTA ATT TA & & 27005-27027 \\
\hline & $P$ & CTG CAT ACA ACC GCT ACC GTA TTG GAA & & $26974-27000$ \\
\hline
\end{tabular}

${ }^{a}$ RT-PCR, reverse transcription-polymerase chain reaction; F, forward primer; R, reverse primer; P, probe.

${ }^{b}$ Location based on the severe acute respiratory syndrome-associated coronavirus, Urbani strain (GenBank accession no. AY278741). 
006413) by using the following primers and probe: forward primer 5'-AGATTTGGACCTGCGAGCG-3'; reverse primer 5'-GAGCGGCTGTCTCCACAAGT-3'; probe $5^{\prime}$-TTCTGACCTGAAGGCTCTGCGCG- $3^{\prime}$. The assay reaction was performed identically to that described above except that primer concentrations used were $30 \mu \mathrm{M}$ each. Fluorescence measurements were taken and the threshold cycle $\left(\mathrm{C}_{\mathrm{T}}\right)$ value for each sample was calculated by determining the point at which fluorescence exceeded a threshold limit set at the mean plus 10 standard deviations above the baseline. A test result was considered positive if two or more of the SARS genomic targets showed positive results $\left(\mathrm{C}_{\mathrm{T}} \leq 45\right.$ cycles $)$ and all positive and negative control reactions gave expected values.

Clinical specimens submitted to CDC for SARS-CoV testing that gave positive results were confirmed with a TaqMan real-time RT-PCR assay based on three different primer and probe sets (Table 1). This assay was performed independently in a separate laboratory using newly extracted nucleic acid from a second specimen aliquot. The confirmatory assay used the SuperScript One-Step RT-PCR (Invitrogen Corp., Carlsbad, CA) and the Mx4000 Multiplex Quantitative PCR system (Stratagene, La Jolla, CA).

\section{Synthesis of RNA Transcripts}

Template for the nucleocapsid gene RNA was plasmid DNA (pCRII, Invitrogen Corp.) containing a full-length copy of the open reading frame for the SARS-CoV nucleocapsid gene oriented behind a T7 promoter. The plasmid was linearized by digestion with SpeI. The template for the polymerase RNA was a RT-PCR product generated by using the following primers: Cor-p-F2-T7, 5'-GTAATA CGACTCACTATAGGGCTAACATGCTTAGGATAA TGG-3' and Cor-p-R2, 5'-CCTATTTCTATAGAGACA CTC-3'. Approximately $1 \mu \mathrm{g}$ of RNA from Vero cells infected with SARS-CoV was used in RT-PCR reactions performed by using the SuperScript RT-PCR kit (Invitrogen Corp.) according to the manufacturer's instructions; both templates were purified by phenol-chloroform extraction and ethanol precipitation before being used for in vitro transcription. RNA was synthesized in vitro by using the MegaScript kit (Ambion Inc., Austin, TX) according to the standard protocol. Synthetic RNA was treated with RNase-free DNase before being purified by phenol-chloroform extraction and ethanol precipitation. The concentration of RNA was determined by use of UV spectroscopy. Synthetic RNA was positive sense and 1,369 $\mathrm{nt}$ in length for $\mathrm{N}$ and $325 \mathrm{nt}$ in length for polymerase.

\section{Results}

\section{Real-Time RT-PCR Sensitivity and Reproducibility}

Tenfold serial dilutions of the polymerase and nucleo- capsid RNA transcripts were tested to assess the copy detection limits and dynamic range of our optimized realtime RT-PCR assays. The lower potential limit of detection was approximately 2 transcript copies per reaction for SARS2 and SARS3, and 7.5 copies per reaction for SARS1 (Figure). The confirmatory assays, which employ three different primer and probe sets (N3, $3^{\prime} \mathrm{NTR}$, and $\left.\mathrm{M}\right)$, showed potential limits of detection similar to the SARS2 and SARS3 assays. Strong linear correlations $\left(\mathrm{r}^{2} \geq 0.99\right)$ were obtained between $\mathrm{C}_{\mathrm{T}}$ values and transcript quantity over at least a $6-\log$ range from approximately $10^{2}$ to $10^{7}$

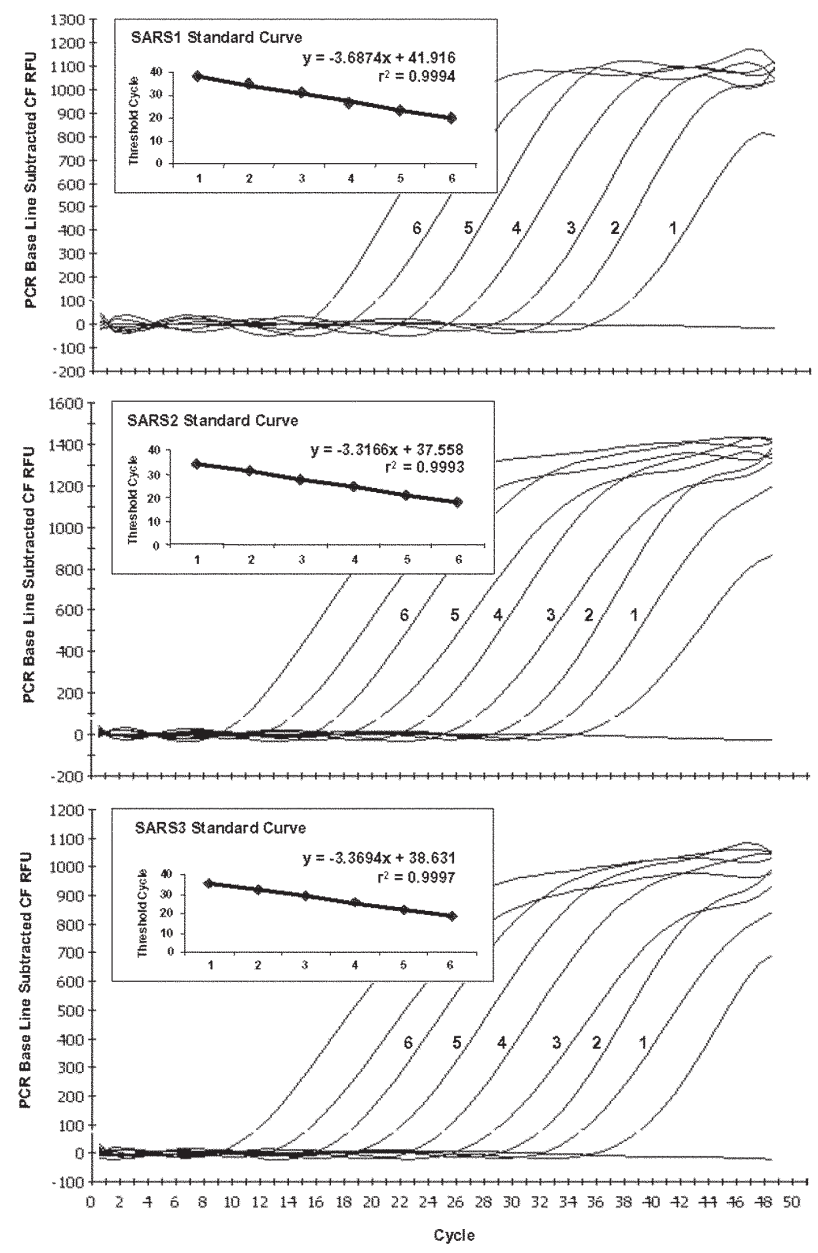

Figure. Typical amplification plot derived from serial 10-fold dilutions of severe acute respiratory syndrome-associated coronavirus RNA transcripts using TaqMan reverse transcription-polymerase chain reaction primer/probe sets SARS1, SARS2, and SARS3. A PCR Base Line Subtractive Curve Fit view of the data is shown with relative fluorescence units (RFU) plotted against cycle number. The default setting of 10 times the standard deviation of fluorescence in all wells over the baseline cycles was used to calculate the threshold cycle, or CT value, for a positive reaction (horizontal line). Inserts show standard curve analysis of the RNA amplification plots with CT values plotted against starting copy number. Plots derived from dilutions containing $2 \times 10^{6}$ to 20 transcript copies for SARS2 and SARS3, and $7.5 \times 10^{6}$ to 75 copies for SARS1. 
copies per reaction for the three primer/probe sets. Linearity was markedly reduced for copy numbers exceeding $10^{6}$ (data not shown).

Assay reproducibility was tested by using replicate 10fold serial dilutions of the RNA transcripts and intra- and interassay variability evaluated for each dilution point in triplicate on three different days. At the lower copy detection limit for SARS2 and SARS3 (2 copies per reaction), assay reproducibility exceeded $90 \%$. In contrast, the lower copy detection limit for SARS1 (7.5 copies per reaction) was positive in $<50 \%$ of replicate reactions. One hundred percent reproducibility with SARS1 was achieved at the dilution that contained 75 transcript copies per reaction. Over the linear range of the assay, the coefficient of variation of the mean $\mathrm{C}_{\mathrm{T}}$ values within and between runs was $0.46 \%-2.54 \%$ and $0.64 \%-2.39 \%$, respectively (Table 2 ).

To assess the efficiency of amplification of the RNA transcripts in the presence of exogenous nucleic acid and potential RT-PCR inhibitors, 10-fold serial dilutions of the RNA transcripts were prepared in water and pooled total nucleic acid extract from 20 SARS-CoV-negative human respiratory specimens (nasopharyngeal aspirates, bronchial washes, sputum, naso- and oropharyngeal swabs, and lung tissue). Exogenous nucleic acid had no discernible effect on amplification efficiency of the SARS1 and SARS3 primer/probe sets, as demonstrated by the similarity in linear regression slopes and endpoint detection limits in the presence and absence of specimen extract (Table 3). In contrast, the standard curve for SARS2 had a more efficient slope $(-3.21)$ in water than in the presence of spiked extract $(-3.48)$ and with greater variation in the $C_{T}$ values at 20 target copies or lower, suggesting that the amplification reaction was less efficient in the presence of the specimen extract. This observation was confirmed on two additional repetitions of the same experiment.

The real-time RT-PCR assay was compared with a previously described conventional RT-PCR for SARS-CoV by using fluorescent dye-labeled primers and GeneScan amplicon analysis (5). Tenfold serial dilutions of a pretitrated SARS-CoV stock adjusted to $1 \times 10^{7} \mathrm{PFUs} / \mathrm{mL}$ were prepared in triplicate and tested by all assays (Table 4). The real-time RT-PCR assays were positive with $100 \%$ frequency at a $10^{-8}$ dilution. Accordingly, the lowest virus quantity detected was $0.01 \mathrm{PFU} / 100 \mu \mathrm{L}$ of specimen extract. The conventional RT-PCR assay was at least 10fold less sensitive in repeat comparisons.

\section{Specificity}

We compared our primer and probe sets with sequences for 14 SARS-CoV field isolates that became available during the course of this study (16) and found no nucleotide mismatches. In contrast, alignments with other published human and animal coronaviruses (GenBank accession no.: human coronaviruses X69721 and AF124989; bovine coronaviruses NC003045 and AF124985; murine hepatitis viruses NC001846 and M55148; sialodacryoadenitis virus AF124990; canine coronavirus AF124986; feline infectious peritonitis virus AF124987; porcine hemagglutinating encephalomyelitis virus AF124988, Z34093, and AF124992; turkey coronavirus AF124991; and avian infectious bronchitis virus NC_001451) showed little sequence identity with our primer and probe sets. To further assess the potential for crossreactions with other members of the Coronaviridae family, the RT-PCR assays were tested against nucleic acid extracts of human respiratory coronaviruses OC43 (VR-759) and 229E (VR-740), feline infectious peritonitis virus (VR-3004), mouse hepatitis virus (VR-1426), bovine coronavirus (VR-874), porcine transmissible gastroenteritis virus (VR-743), and avian infectious bronchitis virus (VR-841), obtained from the American Type Culture Collection (Manassas, VA), and human enteric coronavirus (VR-1475). In addition, nucleic acid extracts of field isolates of influenza A and B; parainfluenza 1,2, and 3; rhinovirus; adenovirus; human metapnuemovirus; and respiratory syncytial virus, as well

\begin{tabular}{|c|c|c|c|c|c|c|}
\hline \multirow[b]{3}{*}{ SARS1 } & \multicolumn{6}{|c|}{ RNA transcript copy number ${ }^{b}$} \\
\hline & $7.5 \times 10^{1}$ & $7.5 \times 10^{2}$ & $7.5 \times 10^{3}$ & $7.5 \times 10^{4}$ & $7.5 \times 10^{5}$ & $7.5 \times 10^{6}$ \\
\hline & & & & & & \\
\hline \multirow{3}{*}{$\begin{array}{l}\text { CV within assay }(\%)^{\mathrm{c}} \\
\mathrm{CV} \text { between assays }(\%)^{\mathrm{d}}\end{array}$} & 2.53 & 0.96 & 0.49 & 0.69 & 1.66 & 0.7 \\
\hline & 2.39 & 1.09 & 0.82 & 0.64 & 2.1 & 0.79 \\
\hline & $2.0 \times 10^{1}$ & $2 \times 10^{2}$ & $2 \times 10^{3}$ & $2 \times 10^{4}$ & $2 \times 10^{5}$ & $2 \times 10^{6}$ \\
\hline \multicolumn{7}{|l|}{ SARS2 } \\
\hline CV within assay (\%) & 1.27 & 0.57 & 0.46 & 0.72 & 0.84 & 0.67 \\
\hline CV between assays (\%) & 1.54 & 1.18 & 0.93 & 1.47 & 1.54 & 1.32 \\
\hline \multicolumn{7}{|l|}{ SARS3 } \\
\hline CV within assay (\%) & 0.8 & 0.55 & 0.65 & 0.5 & 0.27 & 1.25 \\
\hline CV between assays (\%) & 0.94 & 0.64 & 1.07 & 1.13 & 1.24 & 1.65 \\
\hline
\end{tabular}

${ }^{\mathrm{a}} \mathrm{RT}$-PCR, reverse transcription-polymerase chain reaction; $\mathrm{CV}$, coefficient of variation.

${ }^{\mathrm{b}}$ Ten-fold dilutions of the polymerase and nucleocapsid RNA transcripts; copies per reaction; dilution series thawed on 3 different days and assays performed in triplicate for each dilution.

${ }^{c}$ Determined from three replicates within each assay.

${ }^{\mathrm{d}}$ Determined from three independent assays performed on different days. 
Table 3. Efficiency of real-time PCR assays

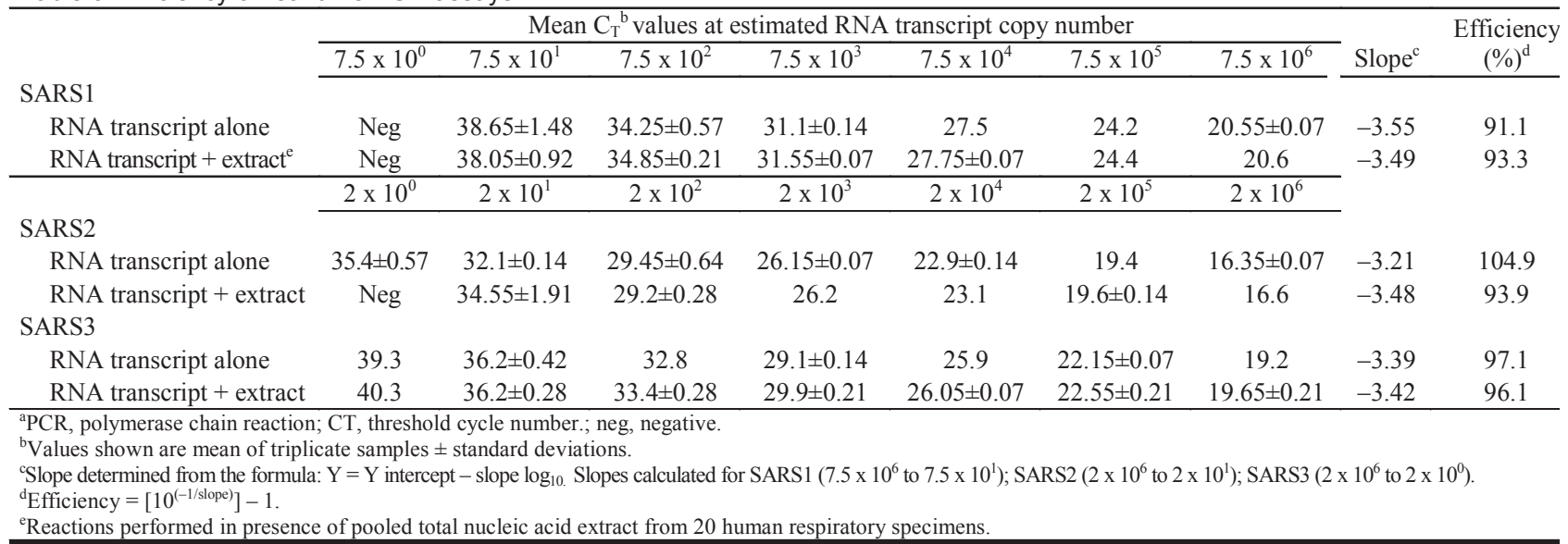

as human and nonhuman primate cell lines were tested. No positive reactions were obtained with any of the primer and probe sets.

\section{Evaluation with Clinical Specimens}

The real-time RT-PCR assay was used to test 14 clinical specimens (including throat swab [2 specimens], sputum [1 specimen], throat wash [5 specimens], and lung autopsy tissues [6 specimens]) from 10 patients with laboratory confirmed SARS-CoV infection (Table 5). Assay results were positive with all specimens for all three primer/probe sets. An additional, 326 respiratory specimens collected during the course of the outbreak from 236 suspected U.S. SARS patients who were serologically negative for SARS-CoV infection were also negative by the real-time RT-PCR.

\section{Discussion}

In response to the SARS outbreak, we developed a realtime RT-PCR assay based on multiple primer and probe sets designed to different genomic targets to facilitate sensitive and specific detection of SARS-CoV in clinical specimens. A potential detection limit of $<10$ transcript copies per reaction was achieved with greater relative sen-

Table 4. Comparison of real-time RT-PCR assay with culture and conventional RT-PCR ${ }^{\mathrm{a}}$

\begin{tabular}{|c|c|c|c|c|}
\hline \multirow{2}{*}{$\begin{array}{l}\text { SARS-CoV } \\
\text { dilution }^{\text {b }}\end{array}$} & \multirow{2}{*}{$\begin{array}{c}\text { Conventional } \\
\text { RT-PCR }\end{array}$} & \multicolumn{3}{|c|}{ Real-time RT-PCR } \\
\hline & & SARS1 & SARS2 & SARS3 \\
\hline $10^{-4}$ & $3 / 3^{\mathrm{c}}$ & $3 / 3$ & $3 / 3$ & $3 / 3$ \\
\hline $10^{-5}$ & $3 / 3$ & $3 / 3$ & $3 / 3$ & $3 / 3$ \\
\hline $10^{-6}$ & $3 / 3$ & $3 / 3$ & $3 / 3$ & $3 / 3$ \\
\hline $10^{-7}$ & $3 / 3$ & $3 / 3$ & $3 / 3$ & $3 / 3$ \\
\hline $10^{-8}$ & $0 / 3$ & $3 / 3$ & $3 / 3$ & $3 / 3$ \\
\hline $10^{-9}$ & $0 / 3$ & $0 / 3$ & $1 / 3$ & $0 / 3$ \\
\hline $10^{-10}$ & $0 / 3$ & $0 / 3$ & $0 / 3$ & $0 / 3$ \\
\hline
\end{tabular}

sitivity than cell culture isolation or conventional RT-PCR. The potential for quantitation over a wide dynamic range (at least 6 logs) was demonstrated with low intra- and interassay variability and limited inhibition from exogenous nucleic acid extract from respiratory secretions. The increased sensitivity of the real-time RT-PCR assay over cell culture and conventional RT-PCR methods may aid detection of the virus at earlier stages of infection, when the virus is present at low titer in respiratory secretions (8). In addition, by eliminating the need for postamplification product processing, the real-time RT-PCR format permitted shortened turnaround time for reporting results, which proved critical during the SARS outbreak.

Although real-time RT-PCR offers clear advantages over more conventional RT-PCR formats, assay results must still be interpreted with caution. For example, the effectiveness of RT-PCR for detection of SARS-CoV in clinical specimens has been shown to be greatly influenced by the quantity, type, and timing of specimen collection $(8,17)$. False-negative results due to poor quality nucleic acid or presence of RT-PCR inhibitors can also be a concern. We addressed this by simultaneously testing for the human RNase P gene, which should be present in all adequately collected samples. False-negative results could also potentially arise from mutations occurring in the primer and probe target regions in the SARS-CoV genome. We addressed this by including multiple genetic targets in our assay and by carefully comparing our primer and probe sequences against published sequences of SARS-CoV as they became available. To avoid falsepositive results, meticulous care was taken to prevent introduction of contaminating viral RNA or previously amplified DNA during preparation of the nucleic acid extracts and amplification reactions. In addition, all RTPCR-positive specimens were retested from a second, unopened sample aliquot and confirmed in a second laboratory by using a real-time assay based on different genetic targets. 
EMERGENCE OF SARS

Table 5. Results of real-time RT-PCR assay with specimens from patients with laboratory-confirmed SARS-CoV infection

\begin{tabular}{lcccccccccc}
\hline & & & & & Vero E6 & Conventional & \multicolumn{3}{c}{ Real-time RT-PCR C ${ }_{\mathrm{T}}^{\mathrm{c}}$ values } \\
\cline { 7 - 10 } Case ID & Location & Specimen ID & Specimen & Serology & culture & RT-PCR $^{\mathrm{b}}$ & SARS1 & SARS2 & SARS3 & RNase P \\
\hline 05078 & Toronto & 2003756523 & Lung, RM & N/A & - & + & 24.2 & 21.6 & 23 & 23.9 \\
& & 2003756525 & Lung, RU & & - & + & 24.9 & 21.5 & 23 & 23.7 \\
05077 & Thailand & 2003756502 & Throat swab & + & + & + & 37.5 & 36.2 & 39.8 & 29.3 \\
05000 & Hong Kong & 2003757035 & Lung, RU & + & - & + & 26.7 & 22.6 & 24.1 & 24.7 \\
& & 2003757036 & Lung, LU & & - & + & 27.2 & 24.9 & 26.5 & 26 \\
& & 2003757037 & Lung, RM & & - & + & 34.9 & 37.5 & 31.9 & 27.4 \\
00220 & Utah, USA & 2003757038 & Lung, LL & & - & + & 29.6 & 27 & 28.6 & 24.5 \\
05001 & Vietnam & 2003757508 & Sputum & + & + & + & 24.7 & 23 & 24.8 & 30.6 \\
05008 & Vietnam & 2003757229 & Throat wash & + & - & + & 23.7 & 22.4 & 24.1 & 30.1 \\
05010 & Vietnam & 2003757239 & Throat wash & + & - & + & 31.1 & 29.3 & 31.5 & 34.2 \\
05013 & Vietnam & 2003757251 & Throat wash & + & - & + & 29.5 & 28.4 & 30.3 & 28.8 \\
05017 & Vietnam & 2003757268 & Throat wash & + & + & + & 26 & 24.7 & 26.4 & 27.9 \\
05316 & Vietnam & 2003759760 & Throat swab & N/A & + & N/A & 25 & 25.3 & 28.2 & 28 \\
\hline
\end{tabular}

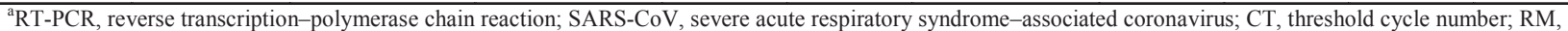
right middle; RU, right upper; LU, left upper; LL, left lower; N/A, not applicable.

${ }^{\mathrm{b}}$ Ref. 5 .

${ }^{\mathrm{c}}$ Values shown mean of duplicate values.

In conclusion, our real-time RT-PCR assay permitted rapid, sensitive, and specific detection of SARS-CoV in clinical specimens and provided needed diagnostic support during the recent SARS outbreak. Widely deploying this assay through the LRN will enhance our ability to provide a rapid response in the event of the possible return of SARS.

\section{Acknowledgments}

We are indebted to Le Thi Quynh Mai, Nghiem Kim Ha, Nguyen Le Khang Hang, Nguyen Thi Hong Hang, Hoang Thuy Long, and the staff of the National Institute of Hygiene and Epidemiology, Hanoi, Vietnam, who provided some of the serum and respiratory specimens used in this study. We also thank James Luby for providing the human enteric coronavirus used in our study.

Shannon Emery is currently earning her Master of Science in Epidemiology from Emory University and working as a guest researcher at the Centers of Disease Control and Prevention in Atlanta, GA. Her research interests include developing real-time PCR platforms for other human respiratory viruses including respiratory syncytial virus and human metapnuemovirus.

\section{References}

1. Poutanen SM, Low DE, Henry B, Finkelstein S, Rose D, Green K, et al. Identification of severe acute respiratory syndrome in Canada. N Engl J Med 2003;348:1948-51.

2. Update: outbreak of severe acute respiratory syndrome-worldwide, 2003. MMWR Morb Mortal Wkly Rep 2003;52:241-8.

3. Lee N, Hui D, Wu A, Chan P, Cameron P, Joynt GM, et al. A major outbreak of severe acute respiratory syndrome in Hong Kong. N Engl J Med 2003;348:1986-94.

4. Tsang KW, Ho PL, Ooi GC, Yee WK, Wang T, Chan-Yeung M, et al. A cluster of cases of severe acute respiratory syndrome in Hong Kong. N Engl J Med 2003;348:1977-85.

5. Ksiazek TG, Erdman D, Goldsmith CS, Zaki SR, Peret T, Emery S, et al. A novel coronavirus associated with severe acute respiratory syndrome. N Engl J Med 2003;348:1953-66.

6. Drosten C, Gunther S, Preiser W, van der Werf S, Brodt HR, Becker $\mathrm{S}$, et al. Identification of a novel coronavirus in patients with severe acute respiratory syndrome. N Engl J Med 2003;348:1967-76.

7. Severe acute respiratory syndrome (SARS): laboratory diagnostic tests [monograph on the Internet]. World Health Organization; 2003 [cited 29 April 2003]. Available from: URL: http://www.who.int/ csr/sars/diagnostictests/en/

8. Peiris JS, Chu CM, Cheng VC, Chan KS, Hung IFN, Poon LLM, et al. Clinical progression and viral load in a community outbreak of coronavirus-associated SARS pneumonia: a prospective study. Lancet 2003;361:1767-72.

9. Mackay IM, Arden KE, Nitsche A. Survey and summary: real-time PCR in virology. Nucleic Acids Res 2002;30:1292-305.

10. Smith IL, Halpin K, Warrilow D, Smith GA. Development of a fluorogenic RT-PCR assay (TaqMan) for the detection of Hendra virus. $\mathrm{J}$ Virol Methods 2001;98:33-40.

11. Lanciotti RS, Kerst AJ, Nasci RS, Godsey MS, Mitchell CJ, Savage $\mathrm{HM}$, et al. Rapid detection of West Nile virus from human clinical specimens, field-collected mosquitoes and avian samples by a TaqMan reverse transcriptase-PCR assay. J Clin Microbiol 2000;38:4066-71.

12. Poon LL, Wong OK, Chan KH, Luk W, Yuen KY, Peiris JS, et al. Rapid diagnosis of a coronavirus associated with severe acute respiratory syndrome (SARS). Clin Chem 2003;49:953-5.

13. Case definitions for surveillance of severe acute respiratory syndrome (SARS) [monograph on the Internet].World Health Organization; 2003 [cited 1 May 2003]. Available from: URL: http://www.who.int/csr/sars/casedefinition/en/

14. Chernesky MA. Traditional serological tests. In: Mahy BWJ, Kangro HO, editors. Virology methods manual. San Diego, CA: Academic Press Inc.; 1996. p. 107-9.

15. Rota PA, Oberste MS, Monroe SS, Nix WA, Campagnoli R, Icenogle JP, et al. Characterization of a novel coronavirus associated with severe acute respiratory syndrome. Science 2003;300:1394-9.

16. Ruan YJ, Wei CL, Ee LA, Vega VB, Thoreau H, Yun STS, et al. Comparative full-length genome sequence analysis of 14 SARS coronavirus isolates and common mutations associated with putative origins of infection. Lancet 2003;361:1779-85.

17. Yam WC, Chan KH, Poon LLM, Guan Y, Yuen KY, Seto WH, et al. Evaluation of reverse transcription-PCR assays for rapid diagnosis of severe acute respiratory syndrome associated with a novel coron- 